\title{
Definitions are important: the case of linear algebra
}

\author{
Abraham Berman 1, Ludmila Shvartsman 2,* \\ ${ }^{1}$ Department of Mathematics, Technion - Israel Institute of Technology, Haifa, Israel \\ 2,* Department of Mathematics, ORT Braude College, Karmiel, Israel \\ For correspondence: berman@tx.technion.ac.il
}

\begin{abstract}
In this paper we describe an experiment in a linear algebra course. The aim of the experiment was to promote the students' understanding of the studied concepts focusing on their definitions. It seems to be a given that students should understand concepts' definitions before working substantially with them. Unfortunately, in many cases they do not. Influenced by their high school experience they concentrate in learning patterns for problem solving without deep understanding of the relevant concepts. In order to educate the students to appreciate the importance of understanding definitions, we changed the homework problems and the didactical contract with the students. We discuss the positive outcomes of these changes from theoretical and educational points of view. We chose to try our approach in a linear algebra course since it is probably the most abstract course for first semester students. We hope that our approach can be useful in teaching other undergraduate courses.
\end{abstract}

Keywords: concept definition, concept image, students' understanding, formal understanding, didactical contract.

\section{Introduction}

Teachers of linear algebra know that the course is difficult for many students. In the words of Stewart and Thomas (2007), students often find their first university linear algebra experience very challenging. Research tells us that one of the main obstacles is the lack of students' understanding of the importance of formal definitions. They try to solve conceptual and procedural problems before they understand the related concepts. This causes distorted concept images which results in misconceptions. We tried to explain to our students that they should change their attitude towards the studied concepts; but, intoxicated with academic freedom, the students stood their ground and ignored the study of definitions.

In this paper we explain how we tried to cope with the students' attitude. We could not change the teaching design of the course since the syllabus, the order of the topics, the number of lectures and tutorials and the format of the examination are compulsory. We could only change the didactical contract with the students. By didactical contract we mean a system of rules, associating the students and the teacher, for a given piece of knowledge (Brousseau (1997)). To promote the appreciation of definitions we slightly changed the form of homework and the assessment of knowledge. We claim that even these slight changes made the study of linear algebra more meaningful and improved the students' understanding and success.

The structure of the paper is as follows. We start with a survey of the research papers on which our work is based. The educational experiment is described in Section 3 and its results are given in Section 4 . The theoretical and practical outcomes are discussed in the concluding section. 


\section{Theoretical Background}

The core of any mathematical discipline is concepts that are introduced by formal definitions. There are many works in mathematics education research that discuss definitions from different points of view. An overview of this research is given in Leikin and Zaskis (2010). The significant role of students' understanding of a formal concept definition in learning mathematics is described by many mathematical educators including Doromolen and Zaslavsky (2003), Edwards and Ward (2008), Leikin and Winicki-Landman (2000), Vinner (1991), Vinner and Dreyfus (1989). They show that understanding of definitions is a prerequisite to proofs of theorems, constructing examples and problem solving. There are different aspects of concept understanding. Berman, Koichu and Shvartsman (2013) suggested operational understanding of a mathematical concept that includes formal understanding, instrumental understanding, representational understanding, relational understanding and applicational understanding. In this paper we concentrate on formal understanding.

Understanding of formal definitions is strongly connected to students' concept image. Tall and Vinner (1981) defined concept image as follows: „We shall use the term concept image to describe the total cognitive structure that is associated with the concept, which includes all the mental pictures and associated properties and processes. It is built up over the years through experiences of all kinds, changing as the individual meets new stimuli and matures" (p. 152). As an example, for a student the concept image of $A^{-1}$ may consist only of the computation of the matrix. As a result, when he is asked whether a given matrix $B$ is the inverse of a matrix $A$, he may calculate $A^{-1}$ instead of multiplying $A B$.

The notion of concept image in calculus has been studied by several researchers (for instance, Dreyfus and Vinner (1989), Leinhardt et al. (1990), Roh, K. (2008)). Concept image in linear algebra was studied by Wawro et al. (2011). Zandieh and Rasmussen (2010) describe a teaching experiment that occurred in an undergraduate geometry course. During the experiment the students were involved in defining activity that includes creating a concept image by using a concept definition. The authors emphasize that such activity is very important in students' progress from informal to more formal ways of reasoning. For a comprehensive review of the interplay between concept definition and concept image see the book of Alcock and Simpson (2009).

Student's understanding of the formal definition of a concept often differs from the exact mathematical definition. In this regard, Edwards and Ward (2004) show that many undergraduate mathematics students fail to understand the distinction between extracted (everyday language) definitions and mathematical definitions and conclude, therefore, that the special nature of mathematical definitions should be addressed more directly in mathematics courses at all levels.

One of the reasons of the students' difficulties with definitions may be the way of teaching mathematics in high school, which for didactical reasons, is often not done rigorously, e.g. Zaslavsky and Shir (2005), Winicki-Landman and Leikin (2000) and Zazkis and Leikin (2008). Undergraduate students try to transfer their previous learning experience to university studies. This experience is based on their beliefs that they should remember some rules and solve a lot of problems, preferably from previous exams. In their opinion learning definitions or proofs is a waste of time.

There are many research papers on teaching linear algebra. Comprehensive surveys include Dorier (2002), who refers to the DNR principles of Harel (2000), and Dorier and Sierpinska (2001). The formal way of reasoning is particularly important in a linear algebra course that requires more abstract thinking than other mathematical courses studied in the first year of undergraduate studies. The resulted difficulties are described in Dorier et al. (2000): „,...the main criticisms made by the students toward linear algebra concern the use of formalism, the overwhelming amount of new definitions and the lack of connection with what they already know in mathematics. It is quite clear that many students have the feeling of landing on a new planet and are not able to find their way in this new world. On the other hand, teachers usually complain of their students' erratic use of basic tools of 
logic or set theory" (p. 28). To cope with these difficulties the authors describe an educational experiment that took place in France that involved drastic changes in the teaching design of a linear algebra course. The experiment resulted in improvement of the students' final grades. As mentioned in the introduction, we could not make such major changes. The changes that we did make will be described in the next section.

\section{The experiment}

The literature surveyed in the previous section strengthened our decision, based on our teaching experience, to make sure that students properly understand formal definitions. Our research goal was to find how this can be done. The derived questions were:

1. Can the students be educated to appreciate the importance of definitions?

2. Does the understanding of definitions contribute to the students understanding of the studied material?

As mentioned in the introduction we studied these questions by changing the didactical contract with the students. We are happy to report that the answer to both questions is affirmative.

The experiment was done in a linear algebra course, given to first semester electrical and electronics engineering students at the Academic ORT Braude College, in the fall semester of 2014. The second author teaches this course every fall semester so we could compare the results of the experiment with the results of teaching in previous semesters. The experiment was repeated in 2015 in order to be sure that the improvement of the students' achievements was consistent. This time we supplemented the experiment by questionnaires given to the students, in order to study the students' appreciation of the weekly homework assignments and the role of definitions in the understanding of concepts and in problem solving. As mentioned in the introduction, the linear algebra course is difficult for many students. We used the questionnaires to check that the course is also difficult for our students.

The course lasts fourteen weeks of four hours of lecture and two hours of tutorial. The textbook is Berman and Kon (2000). The main topics are geometric vectors, fields, systems of linear equations, vector spaces, linear transformations, matrices and determinants, matrix of a linear transformation, eigenvalues and eigenvectors of an operator (a matrix), inner product spaces, and the method of least squares.

The first formal concept that the students meet in the course is a field, the field of complex numbers. Students study complex numbers in high school, but the linear algebra course is the first time that they study them as a field. We want them to understand what a field is since we teach systems of linear equations and vector spaces over a general field. Students are not used to think of complex numbers as ordered pairs of real numbers for which addition and multiplication are defined. They are reluctant to extend the high school definition to the more general formal one. Similar problem occurs in the study of vector spaces, when the students cling to the definition of geometric vectors instead of adopting the general definition of a vector. This conflict increases as we progress through the course and students get more and more abstract material.

Before the experiment the final grade was computed by the following formula:

$$
0.7 F+0.25 \max \{F, M\}+0.05 \max \{F, H\},
$$

where $\mathrm{F}$ is the mark of the final exam, $\mathrm{M}$ is the mark of the midterm exam and $\mathrm{H}$ is the average of homework marks. In general, the student's achievements were mediocre. About $60 \%$ of the students did not pass this course and had to take it again.

One remark on the homework assignments. They were given electronically every week. In each work there were 4-5 procedural problems. The answers were checked automatically. Students could see the number of correct answers immediately after the submission and had one chance to resubmit their answers. After the deadline they got a complete feedback on their work.

In the experiment, reported in this paper, we slightly changed the homework problems and the way of assessment. The procedural questions given as homework were replaced by theoretical questions 
on formal concept definitions and their basic properties. The questions were relatively simple and did not require procedural skills. This enabled the students to solve them before the relevant tutorial, where both computational skills and theoretical understanding were developed. The homework assignments also built up the students' confidence and, at the same time, made them learn the studied material immediately after the lectures and not only before the exams. The way of submission and feedback was not changed but the number of questions in each assignment was reduced to 3 . This was done taking into consideration the students learning load.

The following is an example of a homework problem:

Mark the correct statements (there is at least one correct statement):

1) The sequence of vectors $\left\{u_{1}, u_{2}, \ldots, u_{m}\right\} \subset V$ is a basis of a vector space $V$ if and only if $\operatorname{Span}\left\{u_{1}, u_{2}, \ldots, u_{m}\right\}=V$.

2) The sequence of vectors $\left\{u_{1}, u_{2}, \ldots, u_{m}\right\} \subset V$ is a basis of a vector space $V$ if and only if the sequence is linearly independent.

3) The sequence of vectors $\left\{u_{1}, u_{2}, \ldots, u_{m}\right\} \subset V$ is a basic of a vector space $V$ if and only if the sequence is linearly independent and for every vector $u \in V$ the sequence $\left\{u_{1}, u_{2}, \ldots, u_{m}, u\right\} \subset V$ is linearly dependent.

4) The sequence of vectors $\left\{u_{1}, u_{2}, \ldots, u_{m}\right\} \subset V$ is a basis of a vector space $V$ if and only if the sequence is linearly independent and $\operatorname{Span}\left\{u_{1}, u_{2}, \ldots, u_{m}\right\}=V$.

5) The sequence of vectors $\left\{u_{1}, u_{2}, \ldots, u_{m}\right\} \subset V$ is a basis of a vector space $V$ if and only if the zero vector does not belong to the sequence.

Here the correct answers are (3) and (4). The fourth statement is given in order to check that the students know the formal definition and the third statement is given in order to check whether they understand it.

The second change was that the homework assignments, that were optional, became compulsory. Thus the formula for the final grade was changed to

$$
0.7 F+0.25 \max \{F, M\}+0.05 H \text {. }
$$

The fact that the homework grade became a non-optional part of the final grade was emphasized in the weekly e-mail messages announcing that a new assignment is available.

The questionnaires mentioned above were offered on the web to all the students who took linear algebra in 2015. This was done twice; in the middle of the course (MID) and at the end (END). There were five questions:

1) In your opinion, is the linear algebra course difficult?

2) Do the weekly homework assignments help you to study in a systematic way?

3) Do the weekly homework assignments help you to understand the studied concepts?

4) Are definitions important in understanding the studied concepts?

5) Do you review the definitions of the relevant concepts when you solve problems?

For every question there were the three possible answers: 1) absolutely yes; 2) yes; 3) no. The results are described in the next section.

\section{Results}

The small changes had a big impact. They improved the students' attitude towards the learning process. Unlike previous semesters, the students were interested in the details of definitions and theorems. It was noticeable that they became more active in the lectures. They asked more questions and the questions were more serious. The questions in office hours included theoretical questions and not only technical ones.

The changes did not occur immediately. In the beginning of the course in 2014 the students reacted negatively to the theoretical assignments: "We do not get such assignments in Calculus or in Physics, 
why do we need it here?". It did not take long for the students to change their mind. In personal meetings with many students and in e-mail correspondence with them, they told us that the work on theoretical questions on definitions helped them to understand the concepts. Some of the students told us that they changed their way of problem solving. First, they learn definitions and properties of the related concepts and only then they start to solve the problem. The conversations and correspondence with the students suggested that the students can be educated to appreciate the importance of definitions. This is also evidenced by the answers to the 2015 questionnaires. The questionnaires were offered to 85 students and answered by 68 in MID and by 64 in END.

Table 1. Percentage of the answers to the questionnaires.

\begin{tabular}{|c|c|c|c|c|c|c|c|c|c|c|}
\hline & \multicolumn{2}{|c|}{ Question 1 } & \multicolumn{2}{c|}{ Question 2 } & \multicolumn{2}{c|}{ Question 3 } & \multicolumn{2}{c|}{ Question 4 } & \multicolumn{2}{c|}{ Question 5 } \\
\hline & MID & END & MID & END & MID & END & MID & END & MID & END \\
\hline Absolutely yes & $84 \%$ & $83 \%$ & $24 \%$ & $24 \%$ & $32 \%$ & $26 \%$ & $56 \%$ & $56 \%$ & $32 \%$ & $37 \%$ \\
\hline Yes & $16 \%$ & $17 \%$ & $55 \%$ & $50 \%$ & $54 \%$ & $58 \%$ & $42 \%$ & $37 \%$ & $63 \%$ & $59 \%$ \\
\hline No & $0 \%$ & $0 \%$ & $21 \%$ & $26 \%$ & $14 \%$ & $16 \%$ & $2 \%$ & $7 \%$ & $5 \%$ & $4 \%$ \\
\hline
\end{tabular}

The results show that the linear algebra course is very difficult for our students as well and that the homework assignments helped most of them to organize their studies systematically and to understand the theory. From the answers to Questions 4 (Are definitions important in understanding the studied concepts?) and 5 (Do you review the definitions of the relevant concepts when you solve problems?) we can conclude that many students appreciate the role of definitions in the study of theoretical material and in solving problems.

Thus the answer to the first research question is positive. The second question was whether the understanding of definitions contributes to the students understanding of the studied material?

To answer this question we compared the results of the final exam of three groups of students. There were two experimental groups (EG2014, EG2015). Both consisted of a class of 33 students, who took the course in the fall of 2014 and the fall of 2015. The lecturer in the both groups was the same - the second author. The control group (CG) consisted also of 33 students who took the course with the same lecturer in 2013. The control group was chosen so that even the days and hours of the lectures and tutorials were the same as in 2014 and 2015.

The format of the exam and its level of difficulty were the same for all the groups. There were two parts: a basic one and an advanced one. The basic part consisted of four open problems, to which the students had to write the complete solution. One of the problems $(Q)$ had three parts related to a concept: $Q_{1}$ - the formal definition of the concept, $Q_{2}$ - proof of a theorem related to the concept, $Q_{3}$ - computation related to the concept. For example, $Q_{1}$ - define eigenvector and eigenvalue, $Q_{2}$ prove that eigenvectors corresponding to distinct eigenvalues are linearly independent, $Q_{3}$ - find the eigenvalues and eigenvectors of a given matrix. In the advanced part there were five theoretical statements. The students had to mark if the statement is true or false, and to justify their answer. The statements were more difficult than the statements that were given as home-assignments and to solve them students had to properly comprehend the theoretical material. Here is an example:

Mark true or false and explain your answer: If for a given real $4 \times 4$ matrix $A$ and a given real vector $b \in \square^{4}$ the vectors $u_{1}=\left(\begin{array}{llll}4 & 1 & 4 & 1\end{array}\right)^{T}, u_{2}=\left(\begin{array}{llll}2 & -1 & 2 & -1\end{array}\right)^{T}$ are solutions of the system $A x=b$ of linear equations, then the vector $u=\left(\begin{array}{llll}1 & 1 & 1 & 1\end{array}\right)^{T}$ is an eigenvector of the matrix A corresponding to the eigenvalue $\lambda_{0}=0$.

The following table gives the average results of the final examination. The first row refers to the control group, in which the grade of 70\% of the students was less than 55 . The second row refers to the experimental group in which "only" $40 \%$ of the student did not pass. The G1 is the grade in the 
whole exam, G2 is the grade in the basic part of the exam, G3 is the grade in the advanced part of the exam, G4 is the grade in $Q_{1}$, and G5 is the average grade in $Q_{2}$.

Table 2. Results of the final examinations.

\begin{tabular}{|c|c|c|c|c|c|c|}
\hline Group & $\begin{array}{c}\text { Percent of } \\
\text { failed students }\end{array}$ & G1 & G2 & G3 & G4 & G5 \\
\hline CG & $70 \%$ & 45 & 48 & 34 & 20 & 19 \\
\hline EG2014 & $40 \%$ & 59 & 62 & 47 & 79 & 46 \\
\hline EG2015 & $42 \%$ & 57 & 58 & 54 & 60 & 55 \\
\hline
\end{tabular}

We are pleased to notice that there has also been improvement in the grades in both basic and advanced parts of the exam in 2014 and in 2015 in contrast in 2013. The improvement in G4 is not surprising since learning and understanding of concept definitions was a central part of our teaching practice in 2014 and in 2015.

\section{Discussion}

The paper describes a way to make the study of linear algebra more meaningful. This means that before the students start to solve problems or prove theorems they should understand the related concepts. To achieve that we did two things. We consistently emphasized the importance of understanding definitions and we changed the didactical contract with the students in order to encourage them to learn and understand the concept definitions. Our decision to make the homework obligatory may be considered as not appropriate to university students. We think that for first year students, it was a good decision. There was no similar dilemma concerning the midterm, since in ORT Braude College, the midterm is optional.

The results of our experiment show that students' understanding of formal definitions contributed to their comprehension of the studied material and that their attitude toward studying linear algebra became more serious. It is worthwhile mentioning that the second author also taught a course in calculus to the students in the experimental group took. In the calculus course the teaching philosophy and the didactical contract were not changed and the achievements of the students were lower than their achievements in the linear algebra course. On the other hand, colleagues of us decided to apply our approach in their courses, and in the first semester of 2015 used it teaching discrete mathematics and abstract algebra. The results were very good so it may be interesting to use the "understand concept definitions" in teaching calculus and other course. In discussions with students who came to office hours we observed that as result of what we did, the students' concept images became clearer and more correct. We assume that this is the result of educating the students to appreciate the importance of concept definitions. This assumption cannot be deduced from the tests due to the limitations imposed by their structure and not by the yes/no questionnaires. For this we need in depth interviews with many students. We plan to do it in future research but since we are very pleased with the results of our intervention we decided not to wait with sharing our experiment with other researchers and teachers.

\section{References}

Alcock, L. and Simpson, A. (2009). Ideas from Mathematics Education: An Introduction for Mathematicians. Birmingham, England: Higher Education Academy.

Berman, A., Koichu, B., and L. Shvartsman (2013). The Undersdanding Understanding Equivalnce of Matrices, Proceedings of CERME 8, Antalya, Turkey, 2296-2305.

Berman, A. and Kon, B.-Z. (2000). Linear algebra. Haifa, Israel: BAK (in Hebrew).

Brousseau, G. (1997). Theory of Didactical Situations in Mathematics, Dordrecht, Netherlands: Kluwer Academic Publisher.

Dorier, J.-L. (2002). Teaching Linear Algebra at University. Proceedings of ICM, III(1-3), 875-884.

Dorier, J.-L., Robert, A., Robinet, J., and Rogalski, M., (2000). On a research program about the teaching and learning of linear algebra in first year of French science university. International Journal of Mathematical Education in Sciences and Technology, 31(1), 27-35.

Dorier, J.-L. and Sierpinska, A., (2001). Research into the Teaching and Learning of Linear Algebra, In D. Holton, M. Artigue, U. Krichgraber, J. Hillel, M. Niss and A. Schoenfeld (Eds.), The teaching and Learning of Mathematics at University Level: An ICMI Study (pp. 255-273). Dordrecht, Netherlands: Kluwer Academic Publisher. 
Doromolen, J.V. and Zaslavsky, O., (2003). The many facets of a definition: The case of periodicity, Journal of Mathematical Behavior, 22(1), 91-106.

Edwards, B. and Ward, M.B., (2004). Surprises from Mathematics Education Research: Student (Mis)use of Mathematical Definitions, The American Mathematical Monthly, 111(5), 411-424.

Edwards, B., and Ward, M.B., (2008). The Role of Mathematical Definitions in Mathematics and in Undergraduate Mathematics Courses. In M. P. Carlson and C. Rasmussen (Eds.), Making the Connection: Research and Teaching in Undergraduate Mathematics Education, (pp. 223-232). MAA.

Harel, G., (2000). Three principles of learning and teaching mathematics: Particular reference to linear algebra - Old and new observations. In J.-L. Dorier (Ed.), On the teaching of linear algebra (pp. 177-190). Dordrecht, Netherlands: Kluwer Academic Publisher.

Leikin, R. and Zaskis, R., (2010). On the content-dependence of prospective teachers' knowledge: a case of exemplifying definitions. International Journal of Mathematics Education in Science and Technology, 41(4), 451-466.

Leinhardt, G., Zaslavsky, O., and Stein, M.K., (1990). Functions, Graphs, and Graphing: Tasks, Learning, and Teaching. Review of Educational Research, 60(1), 1-64.

Roh, K., (2008). Students' images and their understanding of definitions of the limit of a sequence. Educational Studies in Mathematics, 69, 217-233.

Stewart, S. and Thomas, M., (2007). Embodied, symbolic and formal thinking in linear algebra. International Journal of Mathematical Education in Science and Technology, 38(7), 927-937.

Tall, D. and Vinner, Sh., (1981). Concept Image and Concept Definition in Mathematics with particular reference to Limits and Continuity. Educational Studies in Mathematics, 12, 151-169.

Vinner, Sh., (1991). The Role of Definitions in the Teaching and Learning of Mathematics. In D. Tall (Ed.), Advanced Mathematical Thinking, (pp. 65-81). Dordrecht, Netherlands: Kluwer Academic Publisher.

Vinner, Sh. and Dreyfus, T., (1989). Images and Definitions for the Concept of function. Journal for Research in Mathematics Education, 20(4), 356-366.

Wawro, M., Sweeney, G., and Rabin, J. M., (2011). Subspace in linear algebra: Investigating students' concept images and interactions with the formal definition. Educational Studies in Mathematics, 78(1), 1-19.

Winicki-Landman, G. and Leikin, R., (2000) On Equivalent and Non-Equivalent Definitions: Part 1. For the Learning of Mathematics, 20(1), 17-21.

Zandieh, M. and Rasmussen, C., (2010). Defining as a mathematical activity: A framework for characterizing progress from informal to more formal ways of reasoning. The Journal of Mathematical Behavior, 29, 57-75.

Zaslavsky, O. and Shir, K., (2005) Students' Conceptions of a Mathematical Definition. Journal for Research in Mathematics Education, 36 (4), 317-346.

Zazkis, R. and Leikin, R. ,(2008). Exemplifying definitions: a case of a square. Educational Studies in Mathematics, 69, 131-148. 